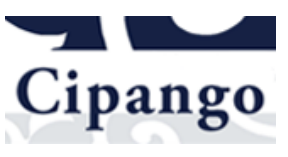

\title{
Cipango
}

Cahiers d'études japonaises

16 | 2009

L'invention des « arts populaires » - Yanagi Sōetsu et le Mingei

\section{Kim Brandt, Kingdom of Beauty: Mingei and the Politics of Folk Art in Imperial Japan (Le royaume de la beauté: le Mingei et la politique de l'art populaire dans le Japon impérial)}

\section{Nadia Sablé-Denans}

\section{OpenEdition}

\section{Journals}

Édition électronique

URL : https://journals.openedition.org/cipango/270

DOI : $10.4000 /$ cipango. 270

ISSN : 2260-7706

Éditeur

INALCO

Édition imprimée

Date de publication : 1 janvier 2009

Pagination : 149-155

ISSN : 1164-5857

Référence électronique

Nadia Sablé-Denans, "KIM BRANDT, KINgDOM OF BEAUTY: MINgEI AND THE POLITICS OF FOLK ART IN IMPERIAL JAPAN (LE ROYAUME DE LA BEAUTÉ : LE MINGEI ET LA POLITIQUE DE L'ART POPULAIRE DANS LE JAPON IMPÉRIAL) ", Cipango [En ligne], 16 | 2009, mis en ligne le 21 novembre 2011, consulté le 30 juin 2021. URL : http://journals.openedition.org/cipango/270 ; DOI : https://doi.org/10.4000/cipango.270

Ce document a été généré automatiquement le 30 juin 2021.

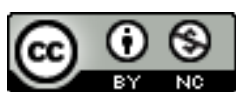

Cipango est mis à disposition selon les termes de la Licence Creative Commons Attribution - Pas d'Utilisation Commerciale 4.0 International. 


\title{
Kim Brandt, Kingdom of Beauty: Mingei and the Politics of Folk Art in Imperial Japan (Le royaume de la beauté : le Mingei et la politique de l'art populaire dans le Japon impérial)
}

\author{
Nadia Sablé-Denans
}

\section{RÉFÉRENCE}

Kim BRANDT, Kingdom of Beauty: Mingei and the Politics of Folk Art in Imperial Japan (Le royaume de la beauté : le Mingei et la politique de l'art populaire dans le Japon impérial), Durham, Duke University Press, 2007, 306 pages. ISBN 978-0-8223-3983-0

1 L'exposition proposée par le Musée du quai Branly en 2008-2009 sur les objets et l'esthétique mingei a donné l'occasion de parcourir les derniers ouvrages parus sur le sujet. La monographie de Kim Brandt, professeure associée en histoire japonaise à l'Université Columbia, mérite sa place parmi ces lectures.

2 Chronologiquement organisé, Kingdom of Beauty expose l'histoire du mouvement pour les Arts populaires de ses origines, dans les années 1920, à la fin de la Seconde Guerre mondiale, en lien étroit avec le processus de construction de la nation japonaise et son expansion coloniale, selon quatre axes politiques et sociaux : l'expansion japonaise en Asie; les idées socialistes dans le monde au début du $\mathrm{xx}^{\mathrm{e}}$ siècle; le fascisme; les mutations de la société japonaise.

3 Cet ouvrage, à contre-courant des publications souvent laudatives qui s'intéressent aux seuls aspects esthétiques des Arts populaires, propose donc une relecture très idéologique et fondamentalement critique de ce mouvement. 
4 Le premier chapitre replace les Arts populaires dans le cadre de la politique coloniale japonaise du début du $\mathrm{xx}^{\mathrm{e}}$ siècle. Il s'agit pour le Japon de créer un «Orient » cimenté par le bouddhisme et d'en prendre la tête afin de s'opposer d'égal à égal à l' " Occident ». Le mingei est ainsi étroitement lié à la présence coloniale en Corée - où Yanagi Sōetsu, personnage central dans la création du mouvement, s'éprend de la céramique Chosŏn dans la seconde moitié des années 1910 -, mais aussi à l'impérialisme occidental en Asie, contre lequel il s'agit de faire bloc.

5 Brandt montre comment le courant des Arts populaires rejette la distinction occidentale entre Beaux-Arts et arts décoratifs et s'appuie sur la tradition japonaise du thé, occupation de l'élite. Toutefois, les tenants du mingei s'en démarquent par un goût pour une céramique d'un style différent, un intérêt non limité aux objets du thé et un refus des critères marchands ou muséologiques dans l'appréciation des pièces. Ils cherchent à imposer un nouveau canon esthétique pour l'appréciation de l'art de la céramique, domaine dominé par l'élite économique bourgeoise.

Grâce à une position présentée comme apolitique, Yanagi obtient en Corée un bâtiment pour son premier musée. Dans le cadre de la politique «culturelle» du pouvoir colonial d'après 1919, cette promotion de l'art coréen encouragée par les autorités est interprétée par Brandt comme visant à maintenir le colonisé dans la position inférieure du génie qui s'ignore car non doué de sens critique. Les adjectifs " triste ", « soumise ", «naïve » attribués à la Corée par le Japon colonial sont à peu près ceux accolés au Japon par l'Occident.

7 Kim Brandt aborde dans le deuxième chapitre la période de mise au point du concept de mingei, marquée par trois étapes: l'intérêt de Yanagi pour le moine-sculpteur Mokujiki shōnin (vers 1923-1926), la guilde de Kamigamo (1927), la Maison des arts populaires (Mingeikan, 1928).

8 Le déplacement de l'intérêt de Yanagi vers les sculptures naïves du moine Mokujiki shōnin ${ }^{1}$ lui permit de rallier à sa cause dignitaires et officiels locaux, collectionneurs et moines bouddhiques. En trois ans, il mit en place un réseau d'intellectuels et de collectionneurs et une revue, avec formation d'un comité et club de lecteurs.

9 Mokujiki shōnin incarnait pour Yanagi la spécificité japonaise face aux autres cultures bouddhiques, mais il ne permettait pas la compétition technique avec l'Occident. C'est pourquoi Yanagi entreprit avec son groupe, dans un contexte général de mode du rural et du non intellectuel, de faire redécouvrir la valeur du savoir-faire artisanal populaire. Il s'éloignait ainsi de l'idée d'une nouvelle culture d'élite et embrassait le progrès social illustré par des objets quotidiens à la fois beaux, fonctionnels, faciles à produire, faits à la main collectivement selon des techniques traditionnelles et peu onéreux. À travers la promotion de ce type d'objet, il s'opposait au culte de l'intellect, de l'individualisme, des machines. Brandt remarque que ces idéaux de gauche, assez communs à cette époque dans la génération de Yanagi, ne firent pas pour autant de lui un marxiste. Il se revendiquait du Guild socialism, courant britannique du début du $\mathrm{xx}^{\mathrm{e}}$ siècle qui prêchait, contre le mode de production industriel, pour un retour à la production artisanale selon le modèle (pseudo) médiéval des guildes. Yanagi était un défenseur de l'inégalité naturelle et appelait de ses vœux une hiérarchie dominée par l'esprit éclairé de l'artiste, de l'universitaire ou du connaisseur. Ceux-ci devaient, par leur appréciation «juste" des produits artisanaux, guider les masses jusqu'au progrès social, avec en 
ligne de mire l'avènement d'un royaume de la beauté universel (et, plus prosaïquement, la promotion de l'« identité japonaise » sur la scène internationale).

La coexistence des techniques traditionnelles et $\mathrm{du}$ fonctionnalisme du design contemporain n'allait pas de soi, mais l'intuition esthétique de Yanagi, parti de l'idée bouddhique de l'art sans ego, se mariait de mieux en mieux avec la condamnation de l'expression romantique du moi par le modernisme naissant et l'idée, déjà consensuelle à l'époque, que le simple est toujours beau.

11 Sur le plan pratique, le programme annoncé dans le pamphlet publié en 1926 en vue de la création de la Maison des arts populaires, qui prévoyait le développement des collections sur trois ans, s'avéra totalement irréaliste. De même, la guilde de Kamigamo, constituée par quelques artisans qui vivaient en communauté afin de réaliser des objets que reconnaîtraient les Arts populaires, fut un échec. Ces objets manufacturés restant chers, elle ne pouvait se maintenir sans subventions.

Le Mingeikan, prototype d'habitation simple conçu pour l'Exposition de promotion de l'industrie nationale organisée à Tōkyō en 1928, eut plus d'impact auprès des riches mécènes que des classes moyennes auxquelles il était destiné. Mais, présenté sous l'angle consensuel de la japonité plutôt que sous celui du progrès social, il fut dans l'ensemble bien accueilli. Il était en accord avec une idée du temps : réintroduire la campagne à la ville (culture du cadeau-souvenir omiyage et de la couleur locale rokaru karā) et la ville à la campagne (démocratisation du tourisme vers le Japon rural). Cette idée était liée au désir des classes moyennes d'avoir accès aux loisirs des classes supérieures, à l'existence de liens familiaux encore forts avec les régions rurales, et constituait une réaction face à la crise économique. Comme les spécialistes des « études folkloriques" (minzokugaku), tel Yanagita Kunio, avec lesquels ils refusaient d'être confondus $^{2}$, les partisans des Arts populaires récusaient le dilettantisme, les uns au nom de la connaissance scientifique, les autres du progrès social. Mais ils ne pouvaient pourtant pas se permettre de tourner le dos aux collectionneurs et aux amateurs, garants du succès de leurs entreprises.

13 Les années trente, abordées plus en détail dans le troisième chapitre, voient apparaître un changement dans la production et la consommation des objets : c'est le «nouvel Art populaire" (shin mingei). Délaissant la collection, ce "nouveau mingei» se propose d'investir les champs du conseil, de la production et du commerce, et s'associe plus volontiers avec les institutions.

L'action sur la production prend la forme d'une entreprise d'amélioration stylistique de l'artisanat des départements de Tottori et de Shimane et d'une introduction d'objets occidentaux dans la fabrication locale (tasses à café, etc.). Il s'agit d'une entreprise de sauvetage esthétique et économique. En effet, outre la satisfaction des façonniers de voir l'artisanat rural de leurs aïeuls apprécié, c'est la crise économique mondiale qui les pousse à coopérer afin de se remettre en selle financièrement.

Bien que Yanagi le déplore, les Arts populaires, au nom de leurs principes esthétiques universalistes, tendent à gommer les particularités régionales. C'est d'autant plus paradoxal que ses partisans pensent se différencier d'autres courants internationaux de soutien à l'art paysan par leur respect des esthétiques régionales et leur exploitation des matières premières locales. Le principal problème au niveau de la production réside dans la difficulté à parvenir à un style de qualité capable de rivaliser avec les antiquités et les œuvres des grands maîtres. Brandt remarque cependant que cette difficulté est 
inhérente au programme, qui cherche justement à mettre sur un pied d'égalité antiquités, artisanat et œuvres d'art.

C'est dans cette phase de son développement que les Arts populaires réussissent à devenir un véritable mouvement. La défection des collectionneurs d'antiquités laisse à Yanagi plus de pouvoir et les régions, dont l'avenir économique est en jeu, s'investissent dans le projet. La production d'objets «mingei » rencontre ses acheteurs, mais les prix prohibitifs et la nature des objets (serre-livres, pommeaux de canne, cravates...) les réservent aux bourgeois urbains et périurbains et, de plus en plus, à leurs épouses.

Finalement, le mingei des années trente est un semi-échec: l'absence de diffusion dans le peuple empêche toute amorce de réforme sociale.

Le quatrième chapitre traite des années quarante et de la collaboration du mouvement des Arts populaires avec l'État japonais, confronté au problème du déficit de la balance commerciale. L'idée des bureaucrates du ministère du Commerce et de l'Industrie est de miser sur l'exportation de produits modernes et japonais. Le recours à la technologie est certes inconciliable avec la promotion des techniques anciennes, mais l'esthétique design des Arts populaires les rend adaptés à ce projet. En outre, ils répondent au goût occidental pour les objets japonisants et font vivre des foyers ruraux.

ailleurs, le gouvernement va voir dans les Arts populaires un ciment de la nation et de l'empire. Deux revues sont ainsi autorisées sans censure. En effet, si la gestion de l'opinion publique en temps de guerre est l'objectif premier de l'État fasciste et ne concerne en rien les Arts populaires, tous deux tendent vers l'avènement d'une « belle société ». Brandt rappelle que les mouvements fascistes italien, allemand ou le gouvernement de Vichy prônaient eux aussi une esthétique et glorifiaient le folklore.

En 1937, un Bureau national de promotion de la région septentrionale du Tōhoku avait demandé à Yanagi des conseils afin d'y organiser une filière artisanale. Lorsqu'aux impératifs économiques de la crise succèdent ceux de la guerre, les intérêts du mouvement des Arts populaires et de l'État convergent une fois encore sans se confondre: la supposée pureté esthétique du Japon des extrémités permet de réintégrer ces régions à la nation, tandis que l'encouragement à acheter japonais pour soutenir l'effort de guerre est une aubaine pour la production mingei. D'un mode de vie « à l'occidentale » réservé à l'élite, on est passé à une culture du quotidien qui induit à la fois la privation solidaire en temps de guerre et l'idée fasciste du conflit nécessaire à l'avènement d'une société nouvelle.

21 Cette dernière position est nettement revendiquée dans la revue Gekkan Mingei, fondée en avril 1939, lorsqu'à l'occasion de la restructuration fasciste des institutions du début des années quarante, les Arts populaires, bien qu'officiellement encouragés, se voient reprocher leur futilité et leur consumérisme. Afin de laver ces soupçons, les responsables du mouvement s'impliquent dans divers projets sociaux parmi lesquels figure la conception, aujourd'hui méconnue, d'un dortoir de travailleuses prenant pour modèle l'architecture paysanne traditionnelle (minka). Il s'agit de rendre les paysannes venues travailler en usine à leur style de vie rural et de préparer par là l'implantation d'usines à la campagne. Sont-ils conscients de chercher à mettre en place un contrôle de type fasciste sur la vie de ces femmes afin d'en faire des travailleuses et des épouses campagnardes modèles? 
Mandchourie. En Chine, les Arts populaires disposaient d'appuis officiels par l'entremise du médecin militaire Yoshida Shōya, lié au mouvement dans sa région de Tottori et ami du rédacteur en chef de la revue Gekkan Mingei, Shikiba Ryūzaburō. Yoshida travailla à Pékin à partir de 1940 pour la Shinminkai / Xinminhui (Société pour le renouveau du peuple), une organisation dépendant du gouvernement provisoire de la Chine du Nord contrôlé par l'armée japonaise, qui était destinée à restaurer l'« esprit asiatique » prétendument souillé par l'influence occidentale. Son idée de délocaliser les Arts populaires en Chine et d'améliorer ainsi l'artisanat local était parallèle à celle de l'armée de délocaliser l'industrie de guerre. Mais le travail de Yoshida s'adressait en priorité aux Japonais et aidait l'armée en soutenant le moral des colons. Il s'agissait surtout de contribuer à la transformation du «mode de vie », ce qui avait toujours été le cœur du projet des Arts populaires.

27 L'ouvrage de Kim Brandt, d'une grande richesse d'informations, éclaire d'un jour nouveau les activités du mouvement des Arts populaires, en particulier au cours des années sombres de la guerre. Il montre notamment de manière inédite comment son discours sur le renouveau de l'art populaire s'étendit en cette période d'expansion coloniale à d'autres pays d'Asie, comme la Corée et la Chine, épousant parfois les orientations idéologiques officielles.

Le style de l'auteur, fluide dans la syntaxe comme dans la logique des enchaînements, permet de donner une image contrastée des Arts populaires. Le déroulement chronologique de l'ouvrage (dernier chapitre excepté) se fait presque sans coupures: 
les réflexions sont tissées au cœur même des énoncés et ne font jamais l'objet de chapitres ou de paragraphes de synthèse. On ne cherchera donc pas dans ce livre des fiches signalétiques des diverses périodes de l'histoire du mouvement, explicites mais figées, mais une description dynamique, multipolaire, riche en questionnements. Ce style particulier aurait été propice à une description du milieu des Arts populaires. En effet, entre les « classes » (lettrées, moyennes, rurales, etc.) et les individus (Yanagi, Yoshida), le groupe des tenants du Mingei reste finalement assez opaque et anonyme. Comment ses membres se connaissaient-ils? Quelles étaient leurs relations intellectuelles et personnelles? Il semble que la forme même du livre de Brandt aurait appelé un traitement approfondi de cette question. De façon plus secondaire, on regrettera la pauvreté et la mauvaise qualité des illustrations et l'absence de certains termes japonais. Mais ces quelques remarques ne gâchent en rien l'intérêt et le plaisir de la lecture de ce Kingdom of Beauty.

\section{NOTES}

1. Voir l'article de François Macé dans ce numéro.

2. Voir la traduction du débat entre Yanagita et Yanagi, dans ce numéro. 